\title{
Magnetotactic Bacteria and Honey Bees: Model Systems for Characterising an Iron Oxide Mediated Magnetoreceptor.
}

\author{
Jeremy Shaw ${ }^{1}$, Alastair Boyd ${ }^{1}$, Michael House ${ }^{2}$, Martin Saunders ${ }^{1}$, Falko Mathes ${ }^{3}$ and Boris Baer ${ }^{4}$. \\ ${ }^{1 .}$ Centre for Microscopy, Characterisation and Analysis, The University of Western Australia, Perth, \\ Australia. \\ 2. Biomagnetics Group, The University of Western Australia, Perth, Australia. \\ 3. School of Earth and Environment, The University of Western Australia, Perth, Australia. \\ 4. Centre for Integrative Bee Research (CIBER), The University of Western Australia, Perth, \\ Australia.
}

Magnetic field perception (magnetoreception) has been described across a broad spectrum of animals, including insects, birds, reptiles and mammals. Despite extensive behavioural evidence demonstrating that these animals are able to sense magnetic fields, the cellular mechanisms involved in transducing a magnetic stimulus to a neuronal response remain a long standing mystery in biology.

The magnetite hypothesis is one possible explanation for how a putative magnetoreceptor might function, and is based on neuronal activation by intracellular nanoparticles of magnetite $\left(\mathrm{Fe}_{3} \mathrm{O}_{4}\right)$ [1]. This is expected to occur when torque is applied to the membrane anchored particles of magnetite in response to changes in Earth-strength magnetic fields. Magnetotactic bacteria provide good evidence for the magnetite hypothesis in animals as they are able to produce chains of magnetite nanoparticles (magnetosomes) in their cytosol (Fig. 1A) and use them for orientation in the Earth's magnetic field.

Finding the cells responsible for magnetoreception has proven to be extremely challenging. The search has been hampered by the fact that only few cells are expected to harbour the sense and they could be located anywhere in the body. Additionally, iron is a widespread biological and environmental element, which can result in contamination of samples and misinterpretation of results [2 and 3]. For these reasons, finding an iron-based magnetoreceptor in situ within an organism or tissue using optical or electron microscopic methods is comparable to the classic needle-in-ahaystack problem. New methodological developments addressing these limitations are timely.

The presence of magnetic particles is a key attribute of magnetoreceptive cells, which can be exploited experimentally in the search for the anatomical location of these cells. Bulk extraction methods that can separate inorganic particles from organic tissue are promising approaches for confirming the presence or absence of magnetite particles.

We used such an approach and developed a novel procedure to extract and concentrate magnetosome particles from bacteria and prepare them for examination using a range of imaging and analytical platforms, including optical, X-ray and electron based microscopy. As a proof of concept, we demonstrate that these particles can be recovered from honey bee abdomen tissue that has been spiked with magnetotactic bacteria (Fig. 1B). Our data show that the magnetosome particles retain their mineral/crystallographic properties after the extraction process, as demonstrated by selected area electron diffraction patterns obtained from undigested and spiked particles (Fig. 1C and Table 1). Our technique was also able to extract iron oxide granules from the honey bee fat body (Fig. 1B). Although these granules are not believed to play a role in magnetoreception, our method can efficiently capture a range of iron materials, which can then be screened for the presence of candidate magnetoreceptor particles. The ultimate aim is to then locate and characterise these particulates in situ [4]. 
References:

[1] J Kirschvink, M Walker and C Diebel, Current Opinion in Neurobiology 11 (2001) pp. 462-467.

[2] C Treiber et al, Nature 484 (2012), pp. 367-370.

[3] N Edelman et al, Proceedings of the National Academy of Sciences 112 (2015) pp. 262-267.

[4] The authors acknowledge funding from the ARC (DE130101660), The University of Western Australia RDA scheme and the Australian Microscopy \& Microanalysis Research Facility (AMMRF).
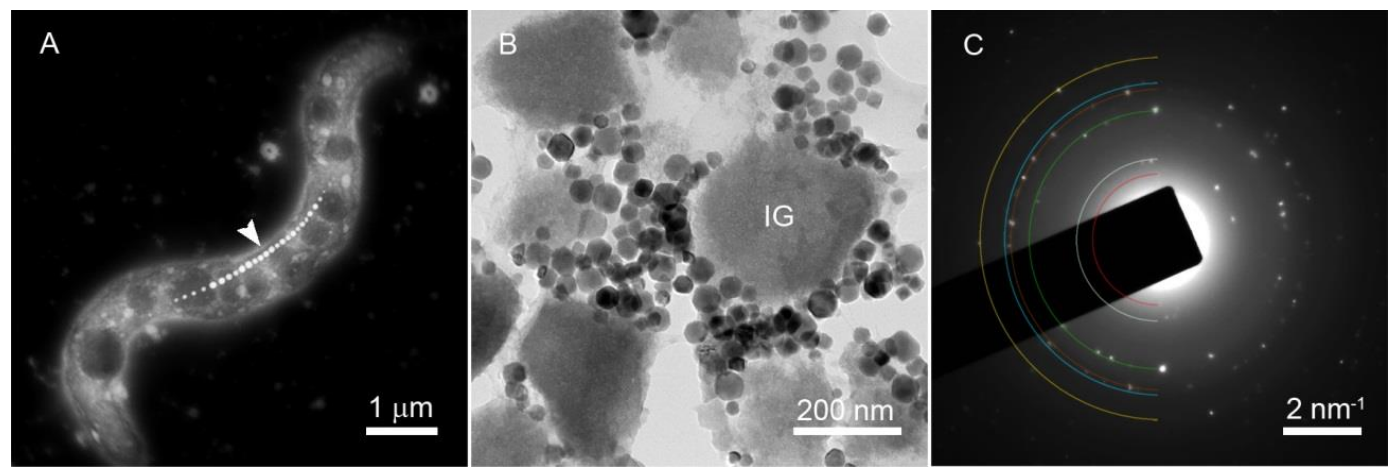

Figure 1. A) Dark field STEM micrograph of a magnetotactic bacterium highlighting the chain of magnetite particles used for orientation (arrowhead). B) Bright-field TEM micrograph of iron granules (IG) extracted from honey bee abdomens using the digestion process. This sample has been spiked with bacterial magnetite particles to demonstrate that they can be recovered during the process and produce C) selected area electron diffraction patterns consistent with those generated from undigested controls. Half-circles denote the rings measured to produce the planar spacings presented in Table 1.

\begin{tabular}{|c|c|c|c|c|c|c|c|c|c|c|c|}
\hline \multirow{2}{*}{$\begin{array}{c}\text { Control } \\
d\end{array}$} & \multirow{2}{*}{$\begin{array}{c}\text { Spiked } \\
d\end{array}$} & \multicolumn{2}{|c|}{ Magnetite } & \multicolumn{2}{|c|}{ Maghemite } & \multicolumn{2}{|c|}{ Hematite } & \multicolumn{2}{|c|}{ Goethite } & \multicolumn{2}{|c|}{ Lepidocrocite } \\
\hline & & $h k l$ & $d$ & $h k l$ & $d$ & $h k l$ & $d$ & $h k l$ & $d$ & $h k l$ & $d$ \\
\hline \multirow{8}{*}{4.90} & \multirow{3}{*}{5.95} & \multirow{8}{*}{111} & - & \multirow{3}{*}{110} & - & & - & \multirow{4}{*}{020} & - & \multirow[t]{7}{*}{200} & 6.20 \\
\hline & & & - & & 5.9 & & - & & - & & - \\
\hline & & & - & & - & & - & & 4.98 & & - \\
\hline & \multirow[t]{5}{*}{4.85} & & 4.85 & 111 & 4.81 & \multirow{5}{*}{012} & - & & - & & - \\
\hline & & & - & & & & - & \multirow[t]{2}{*}{110} & 4.17 & & - \\
\hline & & & - & 210 & 3.73 & & 3.69 & & - & & - \\
\hline & & & - & 211 & 3.40 & & - & 120 & 3.38 & & - \\
\hline & & & - & & - & & - & & - & 210 & 3.28 \\
\hline \multirow[t]{3}{*}{3.02} & \multirow[t]{3}{*}{3.00} & \multirow[t]{3}{*}{220} & 2.97 & 220 & 2.95 & & - & & - & \multirow[t]{3}{*}{101} & 2.97 \\
\hline & & & - & 212 & 2.78 & 104 & 2.70 & 130 & 2.69 & & - \\
\hline & & & - & 310 & 2.64 & & - & & - & & - \\
\hline \multirow[t]{3}{*}{2.58} & \multirow[t]{3}{*}{2.57} & \multirow[t]{3}{*}{311} & 2.53 & 311 & 2.51 & 110 & 2.52 & 021 & 2.58 & & - \\
\hline & & & - & & - & & - & 101 & 2.52 & & - \\
\hline & & & - & & - & & - & 040 & 2.49 & - & - \\
\hline \multirow[t]{2}{*}{2.45} & \multirow[t]{2}{*}{2.45} & \multirow[t]{3}{*}{222} & 2.42 & 222 & 2.41 & & - & 111 & 2.45 & 410 & 2.42 \\
\hline & & & - & & - & & - & 200 & 2.30 & 111 & 2.36 \\
\hline 2.25 & 2.14 & & - & & - & 006 & 2.29 & 121 & 2.25 & & - \\
\hline
\end{tabular}

Table 1. Measured $( \pm 2 \%)$ planar spacings of pre-processed (control) and processed (spiked) particles from magnetotactic bacteria, compared to known spacings of several biogenically relevant iron oxide species, values are in $\AA$. 